\title{
La classification de la diversité de maïs des Mixtèques et des Chatines de la Sierra Sur, Oaxaca Mexique
}

Maize diversity classification by mixtecs and chatinos in the sierra sur, Oaxaca, Mexico

Clasificación de la diversidad del maíz por mixtecos y chatinos en la sierra sur, Oaxaca, México

Quetzalcóatl Orozco-Ramírez et Stephen Brush

\section{OpenEdition}

Journals

Édition électronique

URL : https://journals.openedition.org/ethnoecologie/7319

DOI : 10.4000/ethnoecologie.7319

ISSN : 2267-2419

Éditeur

Laboratoire Éco-anthropologie

Référence électronique

Quetzalcóatl Orozco-Ramírez et Stephen Brush, « La classification de la diversité de maïs des

Mixtèques et des Chatines de la Sierra Sur, Oaxaca Mexique », Revue d'ethnoécologie [En ligne] Supplément 2 | 2021, mis en ligne le 25 novembre 2021, consulté le 20 décembre 2021. URL : http:// journals.openedition.org/ethnoecologie/7319; DOI : https://doi.org/10.4000/ethnoecologie.7319

Ce document a été généré automatiquement le 20 décembre 2021.

Revue d'ethnoécologie est mis à disposition selon les termes de la licence Creative Commons Attribution - Pas d'Utilisation Commerciale - Pas de Modification 4.0 International. 


\section{La classification de la diversité de maïs des Mixtèques et des Chatines de la Sierra Sur, Oaxaca Mexique}

Maize diversity classification by mixtecs and chatinos in the sierra sur, Oaxaca, Mexico

Clasificación de la diversidad del maíz por mixtecos y chatinos en la sierra sur, Oaxaca, México

Quetzalcóatl Orozco-Ramírez et Stephen Brush

\section{Introduction}

L'impact de la culture et de la diversité ethnique sur la diversité et la distinction des populations de maïs a été étudiée dans une zone de la Sierra Sur d'Oaxaca. Les populations de maïs cultivées dans deux municipalités voisines et de groupes ethniques différents (mixtèque vs. chatine ${ }^{2}$ ), se différencient tant morphologiquement que génétiquement, selon une étude fondée sur des collectes d'échantillons, qui a établi des parcelles communes de caractère morphologique et a effectué des analyses des collectes sur des marqueurs génétiques (Orozco-Ramírez et al. 2016). La comparaison des populations de maïs de deux milieux contrastés dans des municipalités montre que les populations de chacune des municipalités ont plus de similitudes génétiques que les populations des milieux similaires, mais issues de groupes ethniques distincts. Cette découverte nous a montré que dans cette région, l'origine sociale (ethnique) a plus d'effets sur la distinction des populations de maïs que les caractères du milieu naturel (ibid). Dans cet article, nous voulons approfondir et comprendre comment les populations de maïs se distinguent par la variabilité culturelle, à travers l'étude des savoirs locaux sur la diversité du maïs. La différence des pratiques agricoles parmi les groupes ethniques a été démontrée dans d'autres régions du pays (Zurita-Benavides 
2016), ce qui permet d'argumenter que la distinction des populations de maïs est également perceptible grâce aux classifications des maïs dans chaque groupe ethnique.

2 La nomenclature locale des variétés a été amplement étudiée dans les principales cultures, et pas seulement pour le maïs (Sadiki et al. 2007). Le nom local d'un type de maïs représente une variété locale, elle est importante en tant qu'unité qui reçoit des pratiques de gestion et, de manière générale, de sélection de la population de maïs (Bellon \& Brush 1994). Le concept de race est actuellement le plus amplement utilisé au Mexique, défini selon Anderson (1946) par les caractères morphologiques de l'épi. Une race est définie comme un ensemble de populations de maïs, dont les caractères communs permettent de les regrouper dans un seul groupe. Jusqu'à maintenant, le nombre des races de maïs au Mexique est de 59 (Sánchez et al. 2000). On peut comprendre une race comme étant l'ensemble des variétés locales. La méthode la plus commune d'étude régionale de la diversité des maïs recommande de commencer par la reconnaissance des variétés locales nommées par les agriculteurs (Hernández-Xolocotzi 1972). On a démontré que la classification des variétés locales de maïs peut surestimer ou sous-estimer autant la diversité morphologique que génétique des maïs d'une localité (Jarvis et al. 2008). La littérature a rapporté que, pour le maïs, les agriculteurs utilisent principalement les caractères du grain et de l'épi pour définir une variété, notamment ceux de la couleur et de la forme (Bellon \& Brush 1994).

3 Berlin (1992) a fondé l'étude de la taxonomie populaire (folk taxonomy) par la description des principes universels qui la régissent. Cependant, cette approche a été récemment critiquée à cause de la grande variation des systèmes traditionnels de classification des organismes; ainsi on a souligné l'importance de la culture dans la structure de ces systèmes de classification (Júnior et al. 2016). C'est par le manque d'études comparatives de la classification locale de la diversité des maïs chez des groupes ethniques voisins partageant un même milieu naturel, que nous avons pensé que ces différences sont dues à la distinction culturelle et, en plus, que ces différences contribuent à maintenir séparées génétiquement les populations du maïs.

4 L'étude des vocabulaires du maïs dans les langues amérindiennes est également importante, car le vocabulaire d'une langue fournit beaucoup d'informations sur les milieux naturels et les cultures locales. On peut en déduire que les concepts qui ont un riche vocabulaire sont plus importants que ceux avec un nombre réduit de mots (Stross 2006). En outre, le vocabulaire du maïs en langue amérindienne a également fourni des données sur la diffusion et la diversification des cultures, grâce à la méthode comparative et à la reconstruction des langues ancestrales, par la linguistique historique (Stross 2006, Hill 2006). Selon ces approches, on sait que les communautés parlant une langue ancestrale issue de la famille Otomangue ont apporté le vocabulaire associé à l'agriculture du maïs (Hopkins 1984, Hill 2006). Une approche similaire peut être utile pour examiner la diffusion et la diversification des types de maïs dans une région particulière. L'étude des noms locaux peut informer sur l'origine des maïs. Par ailleurs, la comparaison des noms partagés démontre les échanges de types de maïs spécifiques.

5 L'objectif de cette publication est de décrire le lien entre la variabilité des maïs de chaque groupe ethnique et la variabilité de la classification locale et des vocabulaires du maïs dans les langues mixtèque et chatine. Nous voulons montrer que, dans ces deux langues, les différences génétiques et morphologiques associées à la différence 
linguistique (voir Orozco-Ramírez et al. 2016) sont liées à la variation des classifications locales du maïs, à la distinction des mots associés au maïs et aux pratiques agricoles.

\section{Le lieu d'étude}

Cette étude a été effectuée dans les municipalités de Santiago Amoltepec et Santa Cruz Zenzotepec. Amoltepec est une municipalité où l'on parle le mixtèque et à Zenzotepec, on parle le chatine. Ces municipalités sont situées à l'ouest de la Sierra Sur d'Oaxaca (Figure 1). Le relief est escarpé, le paysage se caractérise par des montagnes et des ravins. L'altitude va de 105 mètres à 2150 mètres. Les zones basses ont un climat doux et les zones hautes sont tempérées. La température moyenne annuelle est de $26^{\circ} \mathrm{C}$ dans la zone basse et de $18^{\circ} \mathrm{C}$ dans la zone haute. La période des pluies est de mai à octobre, la moyenne de pluies est de 1500 à $2000 \mathrm{~mm}$ annuels (INEGI, Instituto Nacional de Estadística y Geografía, 2013), mais il y a une grande variabilité de l'humidité à cause de l'exposition des versants et de l'altitude. La végétation naturelle est la forêt tropicale (selva baja) dans les zones basses et dans les zones hautes, ce sont des forêts de chênes et de pins. Cependant, la végétation naturelle a été fortement modifiée par l'agriculture et l'élevage.

Figure 1 : Carte de la zone d'étude

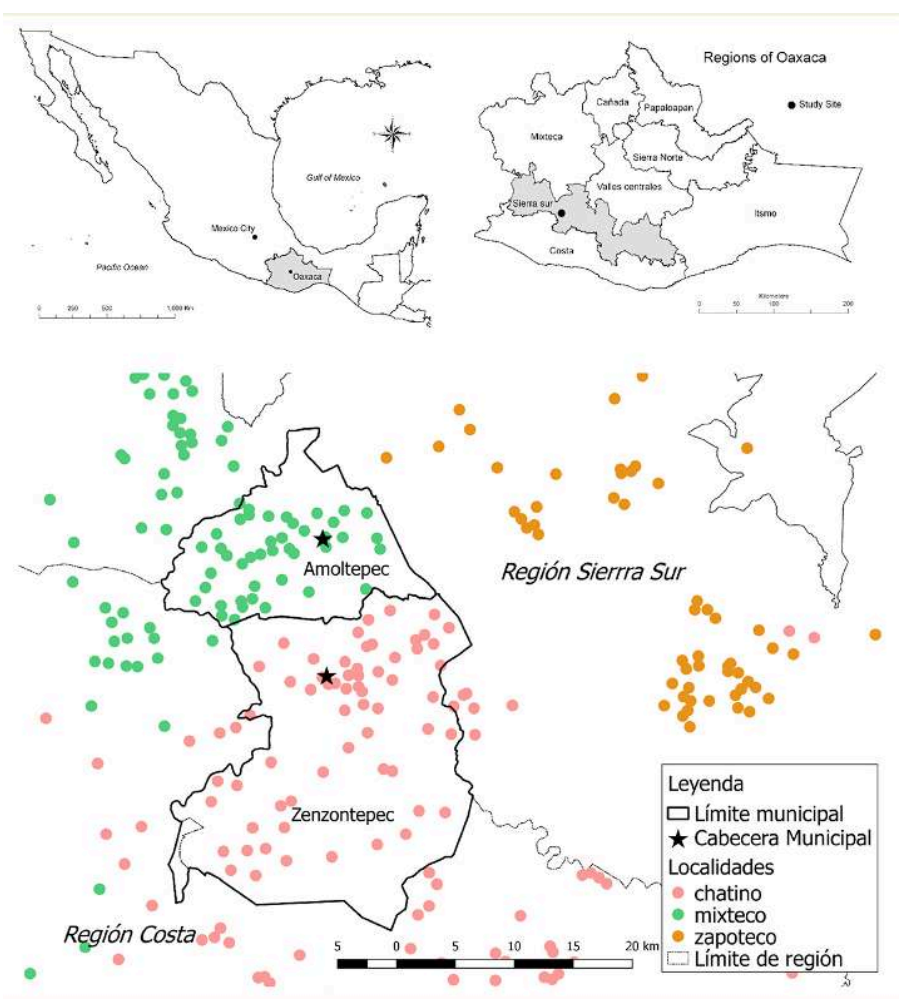

7 La diversité des maiis dans la région de la Sierra Sur est élevée. Aragón Cuevas et al (2012) ont noté 20 races mais ils soulignent aussi les mélanges entre elles, indiquée par la fréquence des gènes, ce sont: mushito, cónico, olotillo, bolita et comiteco. Plusieurs études ont discuté la diversité du maïs dans la région mixtèque (Chávez-Servia et al. 2011) ; la plupart se sont concentrées à Oaxaca sur la diversité morphologique et agronomique (p. ex., Aragón Cuevas et al. 2012, Chávez-Servia et al. 2011). Néanmoins, 
peu d'attention a été portée aux savoirs locaux et linguistiques autour de la diversité des maïs.

400000 personnes environ, parlent le Mixtèque dans les États d'Oaxaca, Puebla et Guerrero, et 50000 environ parlent le Chatine dans le sud d'Oaxaca (INEGI 2010, INALI Instituto Nacional de linguística 2009). La population de Santiago Amoltepec parle le Mixtèque de Santiago Amoltepec, qui est une variante du Mixtèque de la Mixteca Alta (INALI 2009). Les Mixtèques représentent $59 \%$ de la population totale de la municipalité (Tableau 1). La plus grande partie de la population habite dans de petites localités dispersées, la population du chef-lieu ne représente que $10 \%$ de la population totale (INEGI 2010).

Tableau 1 : Population de Santiago Amoltepec et Santa Cruz Zenzontepec

\begin{tabular}{l|c|c|c|c}
\multicolumn{1}{c|}{ Variable } & \multicolumn{2}{c}{ Santiago Amoltepec } & \multicolumn{2}{c}{ Santa Cruz Zenzontepec } \\
\hline Population total & 12,313 & $100 \%$ & 17,897 & $100 \%$ \\
\hline Femmes & 6,383 & $52 \%$ & 9,228 & $52 \%$ \\
\hline Hommes & 5,930 & $48 \%$ & 8,669 & $48 \%$ \\
\hline Moins de 18 ans & 7,011 & $57 \%$ & 9,196 & $51 \%$ \\
\hline Plus de 3 ans qui parle leur langue maternelle & 7,237 & $59 \%$ & 11,132 & $62 \%$ \\
\hline Total de foyers & 2,419 & & 3,587 & \\
\hline Population totale habitant dans la cabecera municipal & 1,263 & $10 \%$ & 1,849 & $10 \%$
\end{tabular}

Zenzontepec est une municipalité plus au nord-ouest de la région chatine. Dans cette municipalité on parle le Chatino occidental alto, parlé également dans certaines localités de Santiago Ixtayutla et San Jacinto Tlacotepec. La population totale de Zenzontepec est supérieure à celle d'Amoltepec, mais les chiffres démographiques sont similaires, comme le pourcentage de la population jeune, qui parle la langue maternelle et vit dispersée dans de petites localités (Table 1). Pour plus d'information sur les caractéristiques culturelles et économiques des municipalités, voir l'étude d'OrozcoRamírez (2014).

\section{Méthodes}

Dans chacune des municipalités, nous avons sélectionné deux localités, l'une située dans les basses terres et l'autre sur les hauteurs de la municipalité, dans le but de montrer la diversité du maïs dans des environnements contrastés. Dans la municipalité mixtèque, nous avons choisi les localités M-L $(598 \mathrm{~m})$ et M-M $(1366 \mathrm{~m})$ et dans la municipalité chatine, Ch-L (518 m) et Ch-M $1121 \mathrm{~m})$. Entre 2011 et 2012, nous avons effectué des entretiens et la collecte d'échantillons de maïs. Avant le début du travail de terrain, nous avons présenté le projet dans les assemblées de chaque communauté (municipalité) pour obtenir les autorisations. De même, chaque agriculteur a reçu par écrit l'information du projet et son consentement pour y participer a été sollicité. Au total, nous avons effectué 86 entretiens, récolté 135 échantillons de maïs, qui ont servi à la classification des races, ainsi que pour les exercices de perception et pour les semer dans les jardins collectifs. Certains échantillons ont été déposés à la banque de germoplasme de l'INIFAP Valles Centrales, et le reste a été donné aux agriculteurs qui ont collaboré à la recherche, pour leur consommation. Au moins $11 \%$ des foyers de chacune des localités ont été interviewés, $12 \%$ en M-M, $30 \%$ en M-L, $14 \%$ Ch-M et $11 \%$ en Ch-L. 
11 Pour chaque échantillon, on a enregistré le nom local, les savoirs agronomiques, les usages et l'origine. La classification par races a été faite par M.C. Flavio Aragón Cuevas, expert des maïs d'Oaxaca. La classification locale des maïs et les savoirs des agriculteurs sur leurs diversités ont été analysés de manière descriptive afin de retrouver les différences et similitudes entre les deux groupes amérindiens. Les vocabulaires du maïs et de l'agriculture ont été analysés pour enquêter sur leurs cognats (parentés). En particulier, on a comparé les noms des parties de la plante avec d'autres vocabulaires des variantes des langues rapportés dans la littérature. Cette analyse comparative est présentée dans le contexte des différences morphologiques et génétiques des populations du maïs de ces groupes amérindiens, décrites par Orozco-Ramírez et al. (2016). Les vocabulaires du maïs ont été enregistrés lors de sessions de travail avec les experts de chaque municipalité ; à Santiago de Amoltepec, cela a été le professeur Margarito García et à Santa Cruz Zenzontepec, le professeur Alfonso Merino, les deux étant reconnus au sein de leur communauté pour leur connaissance de la langue.

\section{Résultats ${ }^{3}$}

\section{Vocabulaire mixtèque et chatine du maïs}

Les deux langues possèdent un vocabulaire riche et diversifié du maïs. Dans la figure 2, nous présentons les noms des principales parties de la plante du maïs dans les deux langues. Le tableau 2 montre le vocabulaire du maïs, incluant les noms locaux des types de maïs, les parties de la plante, les cultures associées, les activités agricoles et certains plats et outils utilisés sur le terrain. Dans les deux langues, on trouve des termes équivalents pour tous les mots enregistrés, sauf pour certains types de maïs qui sont uniques dans chaque région ethnolinguistique. Certains termes non définis s'expliquent par le manque de connaissances des informateurs mais non par l'inexistence des mots.

Figure2 : Noms des parties du maïs en Chatine et en Mixtèque

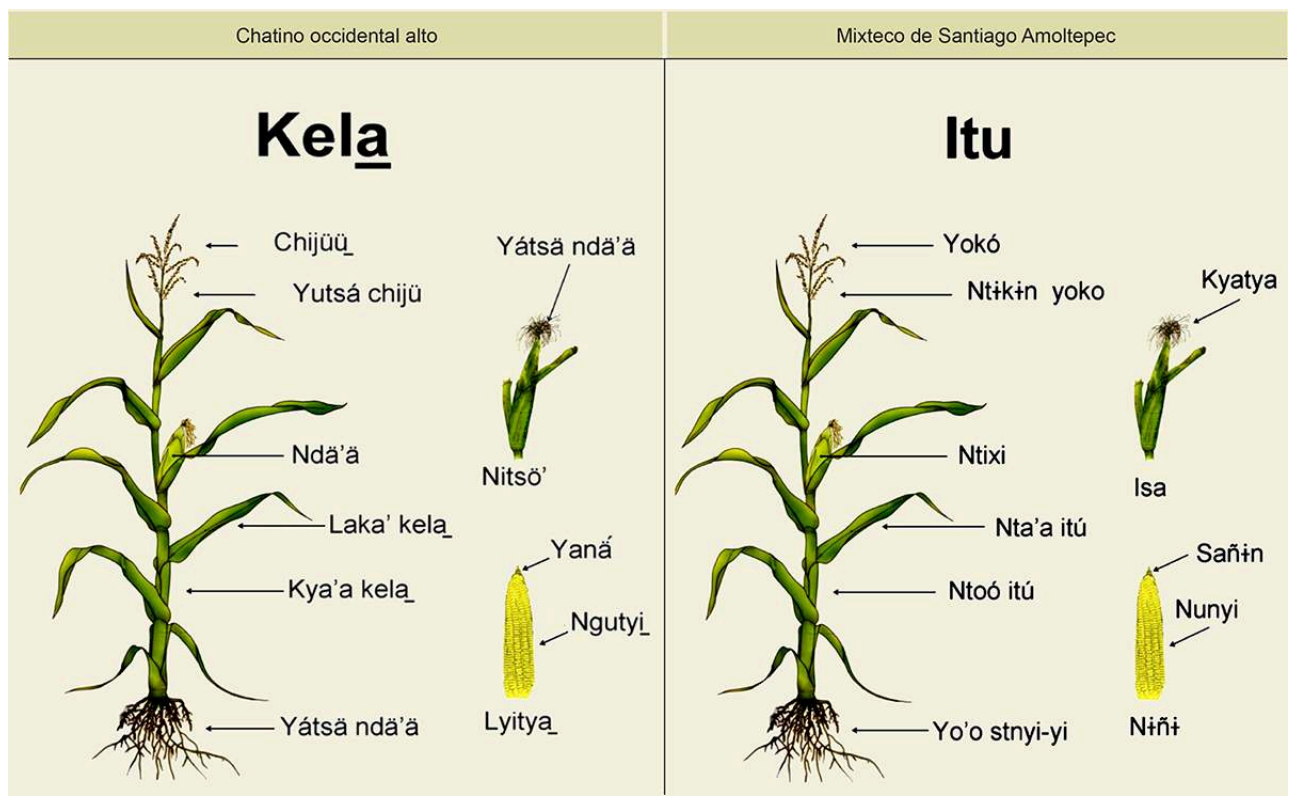


Tableau 2 : Vocabulaire agricole du maïs et des aliments en Chatine et Mixtèque dans les localités étudiées

\begin{tabular}{|c|c|c|c|c|}
\hline Espagnol & $\begin{array}{l}\text { Chatino de } \\
\text { Zenzontepec }\end{array}$ & $\begin{array}{l}\text { Mixteco de } \\
\text { Amoltepec }\end{array}$ & Français & Anglais \\
\hline Abonar & Chu'u yuu lakuti & Sa'a xa'an ñu'u & Fertiliser & Fertilizer \\
\hline Arado & Yarau & Latú & Charrue & Plow \\
\hline Atole & Tyátyikye' & Tuli & Atole (maiis boisson) & Atole (maize drink) \\
\hline Barretón (Barreta) & Barreto & Táka & Bâton fouisseur & Digging stick \\
\hline Cabello de elote & Yátsä ndä'ä & Kyatya & Barbe & Maize silk \\
\hline Calabaza & Chojo & Yikin & Citrouille & Squash \\
\hline Caña de maíz & Kya'a kela & Ntoó itú & Tige de maïs & Maize stalk \\
\hline Campo & Loshë' & $Y_{t^{\prime} t}$ & Terrain & Field \\
\hline Chapulin & Kuityë' ngatsë & Tika & Sauterelle & Cricket \\
\hline Chile & Jnyä' & Ya'a & Piment & Chili \\
\hline Cosecha & Tyu'ú & Skio & Récolte & Harvest (n.) \\
\hline Cosechar & Kisoo' & Naa skio & Moissonner & Harvest (v.) \\
\hline Comal & Jnyä & Chiyó & Comal (tortilla plaque) & Comal (tortilla griddle) \\
\hline Corazón de tortilla & Jlya'ue chaja & Stáa yuu inyi & Coeur de la tortilla & Heart of the tortilla \\
\hline Corte de monte (talar) & Ka'nä & Ta'antya yi’' & Couper la végétation pour préparer le terrain & Cut brush to prepare field \\
\hline Desgranar & Kusukua' & Skoyo nunyi & Séparer le grain de maïs épis & Shuck grain from maize cob \\
\hline Elote & Ndä'ä & Ntixi & Épi de maïs & Young maize ear \\
\hline Embrión & Nu lu'u ji'ti ntsukua' & Se'e nunyi & Embryon & Embryo \\
\hline Empanada & Mbele'nkyá ndoo tyá'a & Staa tichiti & Tarte & Empanada \\
\hline Escardar & & Skaa itú & Sarclage & Weed (v.) \\
\hline Espiga & Chijüü & Yokó & Maïs gland & Tassel \\
\hline Frijol & Ndaa & Ntutyi & Haricot & Beans \\
\hline
\end{tabular}

\begin{tabular}{|c|c|c|c|c|}
\hline Espagnol & $\begin{array}{l}\text { Chatino de } \\
\text { Zenzontepec }\end{array}$ & $\begin{array}{l}\text { Mixteco de } \\
\text { Amoltepec }\end{array}$ & Français & Anglais \\
\hline Gorgojo & Kuako'o & Tikivi & Charançon & Weavil \\
\hline Grano & Ngutyi & Nunyi & Grain & Kernel (grain) \\
\hline Gusano & Ngonu' & Tintaku & Ver & Worm \\
\hline Hoja de milpa & Laka'kela & Nta'a itú & Feuille & Maize leaf \\
\hline Huitlacoche (cacalote) & Kuaye' & tika'aya & Charbon du maîs comestible (Huitlacoche) & Edible maize smut (Huitlacoche) \\
\hline Jilote & Nitsö' & Isa & Maïs épi jeune & Green maize ear \\
\hline Limpia de la siembra & Kinyä jnï & Kutu itú & Sarclage du champs & To clean a field \\
\hline Limpiar & Kinyää & Kutu & Nettoyage champ après la récolte & Field cleanup after harvest \\
\hline Limpiar maiz & Kibii ntsukua' & Ntakachi nunyi & Pour nettoyer le maïs & To clean maize \\
\hline Machete & Majtyi-mashtyi & Mastyi & Machette & Machete \\
\hline Maleza & Kishë' & Ti'vi itu & Mauvaises herbes & Weed (n.) \\
\hline Maíz & Ntsukua' & Nunyi & Maïs & Maize \\
\hline Maíz Amarillo & Ntsukua ngáchị & Nunyi kwan & Maïs jaune & Yellow maize \\
\hline Maíz blanco & Ntsukua ngatë & Nunyi kwichin & Maïs blanc & White maize \\
\hline Maiz chico & Ntsukua lue & Nunyi ntyii' & Petit maïs & Small maize \\
\hline Maiz grande & Ntsukua tunü & Nunyí na'nu & Gros maïs & Large maize \\
\hline Maiz negro & Ntsukua ngatá & Nunyi tnu & Maïs noir & Black maize \\
\hline Maiz nuevo & Ntsukua kukui & Nunyi chaa & Nouveaux maîs & New maize \\
\hline Maiz picado & Ntsukua keta & Nunyi tikixin & Maïs avec charançons & Worm eaten maize \\
\hline Maiz pinto & Ntsukua pintyu & Nunyi pintu & Maïs multicolore & Multi-colored maize \\
\hline Maíz rojo (colorado) & Ntsukua ngá'a & Nunyi kwa'á & Maïs rouge & Red maize \\
\hline
\end{tabular}




\begin{tabular}{|c|c|c|c|c|}
\hline Espagnol & $\begin{array}{c}\text { Chatino de } \\
\text { Zenzontepec }\end{array}$ & $\begin{array}{l}\text { Mixteco de } \\
\text { Amoltepec }\end{array}$ & Français & Anglais \\
\hline Maiz cuarenteño & Ntsukua jnï lue & Nunyi uxiko ntu & Maïs de 40 journées & 40 day maize \\
\hline Maíz del cerro & Ntsukua shi tyikala' & Nunyí yuku & Maïs du collines & Hill land maize \\
\hline Maiz tempranero & Ntsukua jnï jlyala & Takwatyi & Bref campagne de maïs & Short season maize \\
\hline Maíz olotillo & Ntsukua látí yaná & Nunyi tayiki & Olotillo maïs & Olotillo maize \\
\hline Maiz de conejo & Ntsukua Jnï kuichi & Nunyi tata iso & Conejo maïs & Conejo maize \\
\hline Maíz de camarón & Ntsukua jnï kuee' & Nunyi Takwatyi & Maïs de crevettes & Shrimp's maize \\
\hline Maiz chiriqui & & Tayiki chitiki & Mince épi maïs & Thin eared maize \\
\hline Maiz enano & Ntsukua jnï lue & & Maïs nain & Dwarf maize \\
\hline Maíz palmasol & Ntsukua'jnï tyikuë & & Palmasol maïs (Iongue saison) & Palmasol maize (long season, off white) \\
\hline Maiz largo & & Nunyi tata kumi yoó & Longue campagne de maïs & Long season maize \\
\hline Maíz de guajolote & & Nunyi tata ko'lo & Maïs de dinde & Turkey's maize \\
\hline Maiz de tierra caliente & & Nunyi ñuu ka'nyi & Maïs d'endroit chaud & Lowland maize \\
\hline Maíz tardón & & Nunyi tata kumi yoó & Longue campagne de maïs & Late season maize \\
\hline Masa & Sukuä & Yuchan & Pâte de maïs & Meal for tortillas \\
\hline Mazorca & Lyitya & Niñ̈i & Épi de maïs & Maize ear \\
\hline Memela & Mbele' & Xa'antya & Galette de maïs grillé & Toasted maize cake \\
\hline Metate & Kichi & Yosó & Mortier de pierre pour le maïs & Stone mortar for maize \\
\hline Milpa (campo) & Nyä kelạ & & Champ de maïs (milpa) & Mixed crop field (milpa) \\
\hline Milpa (planta) & Kela & Itu & Plante de maïs (maïs) & Maize plant (milpa) \\
\hline Mole (con maíz) & $T i^{\prime} \underline{i}$ & Tutya & Sauce chili (mole) avec le maïs & Chili sauce (mole) with maize \\
\hline Monte & Loshë' & $Y f^{\prime \prime} t^{\prime}$ & Fourré & Brush \\
\hline Red (morral) & Kesu-kuejë kitse & Ñunu ntikin & Musette & Backpack \\
\hline Espagnol & $\begin{array}{c}\text { Chatino de } \\
\text { Zenzontepec }\end{array}$ & $\begin{array}{l}\text { Mixteco de } \\
\text { Amoltepec }\end{array}$ & Français & Anglais \\
\hline Olote & Yaná & Sañin & Rafle & Cob \\
\hline Pinole & Keta ntsukua & Yutyi vixí & Sol maïs rôti avex épices (Pinole) & Roasted ground maize with spices (Pinole) \\
\hline Pizcar & Kisuu lyitya & Skyo & Moissoner & To pick \\
\hline Planta jiloteando & Ndesä'ä nitsö' & Nïi isa itú & Plante avec de jeunes oreilles du maïs & Plant with young maize ears \\
\hline Planta de maiz tierna & Kela kuénë' & Itú yutya & Les jeunes plantes de maïs & Young maize plant \\
\hline Milpa lista para pizcar & Kela ta ngubityi & Chaa nta ityí itú & Champ de maïs prêt pour moissonner & Maize field ready for picking \\
\hline Planta de maíz pequeña & Kela lue & Itú lu'lu & Petite plante du maïs & Small maize plant \\
\hline Polen & Yutsá chijü & ntikin yoko & Pollen & Pollen \\
\hline Poner nixtamal & Ntsukua ngukye' & Styi'yó nunyí & Préparer nixtamal & Prepare nixtamal \\
\hline Pozole & Ntsukua' nkyatsu & Ntucha & Soupe avec du maiis (le pozole) & Soup with maize (pozole) \\
\hline Raiz & Tyëë & Yo'o stnyi-yi & Racine & Roots \\
\hline Rozar & Ka'nä & Kutu yit' & Brûler avant de planter & Burn before planting \\
\hline Sembradio de maiz & Nyää kela & Ñu'u itú & Champ de maïs & Maize field \\
\hline Sembrar & Kuta & Tachi & Semer & To sow \\
\hline Semilla de maíz & Ntsukua jiil & Nunyi tata & Semences de maïs & Maize seed \\
\hline Surco & Leta & Yukún & Rangée & Row \\
\hline Tamal de elote & & Sun & Tamale du jeune maïs & Tamale of young maize \\
\hline Tamal & Chajakuu & Tikoó & Tamale & Tamale \\
\hline Tierra & Yuu & $N u^{\prime} u$ & Terre & Land \\
\hline Tortilla & Chaja & Staá & Tortilla & Tortilla \\
\hline Tostada & Chaja laki’i & Staá tikasun & Tostada & Tostada \\
\hline Totomostle & Uela' & Ñama & Spathes de maïs & Maize husk \\
\hline Troje & Ja'ua & Yaká & Grange de maïs & Maize granary \\
\hline Yunta & & Naa skuto jntikt & Paire de boeufs & Yoke of oxen \\
\hline
\end{tabular}

13 Le vocabulaire contient en tout 89 mots. Seuls quatre mots paraissent de vrais cognats (Hill 2006). Ces mots sont le piment (ya'a en Mixtèque et jnyä' en Chatine), grain (nunyi en Mixtèque et ngutyi en Chatine), maïs (nunyí en Mixtèque et ntsukua' en Chatine) et terre (nu'u en Mixtèque et yuu en Chatine). Ces termes peuvent être des mots très anciens venus de la langue ancestrale, il y a approximativement 4700 ans, quand l'Otomangue de l'est s'est séparé donnant la branche pololoca-zapotèque et l'amuzgomixtèque (Kaufman 1990). Nous retrouvons un autre mot similaire dans les deux langues, mais il s'agit d'un emprunt à l'espagnol, c'est le mot machete : majtyi o mashtyi en Chatine et mastyí en Mixtèque.

14 Quand on compare les douze noms des parties du maïs en langues chatine et mixtèque des communautés voisines, on remarque peu de différences (Tableau 3). Entre le Chatine de Zenzontepec et le Chatine de Tataltepec (village situé à $26 \mathrm{~km}$ au sud de Zenzontepec), on trouve une très légère variation des mots se référant à l'épi et au 
plant de maïs, ainsi qu'une variation plus grande pour la racine (Tableau 3). Entre le Chatine de Zenzontepec et celui de Panixtlahuaca (village situé à $34 \mathrm{~km}$ au sud-est de Zenzontepec), la plus grande divergence a été trouvée pour les mots pollen et racine, mais pour les dix autres mots il n'y a qu'une légère variation dans la prononciation (Tableau 3).

Il n'existe pratiquement pas de différences dans ce groupe de mots entre le Mixtèque de Amoltepec et celui de Yosundúa (village situé à $30 \mathrm{~km}$ au nord d'Amoltepec), à part une exception, les cheveux de l'épi. Dans ces deux langues et le Mixtèque de San Juan Colorado (village situé à $53 \mathrm{~km}$ au sud-est d'Amoltepec), il n'existe qu'une petite variation pour le mot de l'olote, « cœur de l'épi » et totomoxtle 4 : sañin (cœur de l'épi) à Amoltepec et xiñüi à San Juan Colorado; ñama (totomoxtle) à Amoltepec et yama à San Juan Colorado. Ces divergences mineures pour les mots des parties du maïs dans le groupe mixtèque et dans le groupe chatine peuvent suggérer qu'une communication $\mathrm{e}$ eu lieu entre les agriculteurs à propos du maïs dans chaque groupe linguistique. Cela suppose également qu'il peut y avoir plus de facilité pour que les semences circulent dans les groupes mixtèque et chatine, mais pas entre ses deux groupes, même si, géographiquement, ils sont proches.

Tableau 3 : Vocabulaire des parties de la plante du maïs avec les variations linguistiques de la région

\begin{tabular}{|c|c|c|c|c|c|}
\hline Anglais & Français & Espagnol & Chatino de Zenzontepec & Chatino de Tataltepec ${ }^{1}$ & Chatino de Panixtlahuaca ${ }^{2}$ \\
\hline Maize silk & Barbe & Cabello de elote & Yátsä ndä'ä & & \\
\hline Maize stalk & Tige de maïs & Caña de maíz & Kya'a kela & Lijya calya & Lijya kala \\
\hline Young maize ear & Épi de maïs & Elote & Ndä'ä & Nda'a & Nda'an \\
\hline Tassel & Maïs gland & Espiga & Chijüü & & Juun kala \\
\hline Maize leaf & Feuille & Hoja de milpa & Laka'kela & Laka'calya & Lka'kala \\
\hline Green maize ear & Maïs épi jeune & Jilote & Nitsö' & & Katsun' \\
\hline Maize & Maïs & Maíz & Ntsukua' & Nscua' & Nskwa \\
\hline Maize ear & Épi de maîs & Mazorca & Lyityạ & Ltya & Tya \\
\hline Maize plant (milpa) & Plante de maïs (maïs) & Milpa (planta) & Kela & Calya & Kala \\
\hline Cob & Rafle & Olote & Yanấ & & Ynan \\
\hline Pollen & Pollen & Polen & Yutsá chijü & & Kata keé \\
\hline Roots & Racine & Raíz & Tyёё & Quiche suu yaca & Suun \\
\hline Maize husk & Spathes de maïs & Totomostle & Uela' & & Lka'tla' \\
\hline
\end{tabular}

'Pride L. y Pride K. 1970. ${ }^{2}$ Pride K y Pride L. 2010. ${ }^{3}$ Beaty de Farris K. 2004. ${ }^{4}$ Campbell et al. 1986

Le nom local des types de maïs en espagnol est en général traduit littéralement de la langue vernaculaire (mixtèque ou chatine). Par exemple, le maïs rouge en Mixtèque est nunyí kwa'á, nunyí est maïs et kwa'á signifie rouge. En Chatine, c'est ntsukua ngáa á, le second mot signifie rouge. Les noms en langue vernaculaire peuvent nous donner une idée de l'importance et des relations des types de maïs. En Mixtèque, le seul type de maïs qui ait un nom propre est le maïs corto (takwatyi). À la différence des autres noms des maïs, celui-ci ne se nomme pas avec le mot maïs mais avec un adjectif. Nous pouvons déduire que ce type de maïs (takwatyi) est important car il a un nom propre. En Chatine, nous n'avons trouvé aucun nom unique, tous sont dénommés avec le mot maïs et un adjectif. Autre cas intéressant, le maïs olotillo en Mixtèque; le maiis olotillo et le maïs chiriqui ont un même adjectif tayiki, suggérant que les deux types sont similaires pour les agriculteurs.

17 Au total, on enregistre 20 noms de types de maïs en Mixtèque, et 17 en Chatine. Ces chiffres représentent le nombre des types de maïs que l'expert connait en langue 
vernaculaire, mais ils ne couvrent pas tous les noms connus par tous les agriculteurs des communautés. Ce grand nombre de noms montre les savoirs détaillés connus de la diversité des maïs. Nous n'avons pu trouver aucun nom de maïs similaire en Mixtèque ou en Chatine, ceci peut dénoter la séparation culturelle dans la gestion des populations de maïs de chacun des groupes.

\section{Classification locale de la diversité de maïs}

La diversité des maïs est élevée dans les deux municipalités. Nous avons retrouvé sept races : Olotillo ( $37 \%$ sur le total des échantillons dans les deux municipalités), Tuxpeño (35\%), Conejo (16\%), Tepecintle (10\%), Elotes occidentales (0,7 \%), Zapalote grande (0,7 \%) et Pepitilla $(0,7 \%)$. Des trois dernières, seulement un échantillon a été enregistré. La plupart des échantillons collectés ont été des mélanges de deux ou plusieurs races. Seulement 51 des 135 échantillons avaient les caractéristiques dominantes d'une race. Lorsqu'on considère la race principale á laquelle correspond chaque échantillon, nous avons trouvé plus d'échantillons de Conejo et Tepecintle dans les localités mixtèques et plus d'échantillons des races Olotillo et Tuxpeño dans les communautés chatines (Tableau 4). Cette différence est statistiquement significative ( $p=0.0001,1000$ simulations Monte Carlo).

Tableau 4 : Distribution de races primaires de la région, numéros des échantillons collectés dans chaque municipalité

\begin{tabular}{l|c|c|c|c|c|c}
\hline \multirow{2}{*}{ Race primaire } & \multicolumn{2}{|c|}{ Municipalité Mixteco } & \multicolumn{2}{c}{ Municipalité Chatino } & Ch-L \\
\hline & M-M & M-L & Ch-M & 2 & 21 \\
\hline Conejo & 10 & 3 & 6 & 1 & 1 \\
\hline Elotes occidentales & 0 & 0 & 0 & 12 & 50 \\
\hline Olotillo & 10 & 7 & 21 & 0 & 1 \\
\hline Pepitilla & 0 & 0 & 1 & 0 & 14 \\
\hline Tepecintle & 6 & 7 & 1 & 19 & 47 \\
\hline Tuxpeño & 7 & 6 & 15 & 1 & 1 \\
\hline Zapalote grande & 0 & 0 & 0 & 35 & 135 \\
\hline Total & 33 & 23 & 44 & & \\
\hline
\end{tabular}

La classification locale du maïs se base principalement sur les caractères externes de l'épi, les caractéristiques phénologiques et l'origine de la variété. À partir de cette information, on donne des noms locaux à chaque type de maïs. Nous trouvons plus de noms locaux dans les localités chatines que dans les mixtèques : 17 types (noms) en Ch$M$ et 15 en Ch-L, 9 en M-M et 6 en M-L (Tableau 5). 
Tableau 5 : Nom des types de maïs trouvés dans chaque localité et pourcentages des échantillons

\begin{tabular}{|c|c|c|c|c|c|c|c|}
\hline \multicolumn{4}{|c|}{ Chatino } & \multicolumn{4}{|c|}{ Mixteco } \\
\hline \multicolumn{2}{|c|}{ Chatina alta (Ch-M) } & \multicolumn{2}{|c|}{ Chatina baja (Ch-L) } & \multicolumn{2}{|l|}{ Mixteca Alta (M-M) } & \multicolumn{2}{|c|}{ Mixteca baja (M-L) } \\
\hline Olotillo $(0)^{*}$ & $39 \%$ & Maíz de sapo (Tx) & $20 \%$ & Largo $(0)$ & $27 \%$ & Olotillo (O) & $26 \%$ \\
\hline Olotillo corto $(0)$ & $11 \%$ & Olote delgado $(0)$ & $14 \%$ & Corto (C) & $21 \%$ & Corto (Tx) & $22 \%$ \\
\hline Olotillo largo (O) & $9 \%$ & Corto (O) & $9 \%$ & Corto negrito (C) & $15 \%$ & Largo (T) & $22 \%$ \\
\hline Amarillo (C) & $7 \%$ & Corto pinto (Tx) & $9 \%$ & Blanco (O) & $9 \%$ & Blanco $(T)$ & $17 \%$ \\
\hline Cuarenteño $(\mathrm{C})$ & $5 \%$ & Olotillo (O) & $9 \%$ & Amarillo (Tx) & $6 \%$ & Medio olotillo $(\mathrm{O})$ & $9 \%$ \\
\hline Maiz enano $(T x)$ & $5 \%$ & Blanco (Tx) & $6 \%$ & Corto Amarillo (C) & $6 \%$ & Maiz de chocue $(0)$ & $4 \%$ \\
\hline Blanco (O) & $2 \%$ & Maiz de la costa (Tx) & $6 \%$ & Maiz de tierra caliente $(\mathrm{Tx})$ & $6 \%$ & & \\
\hline Conejo (Tx) & $2 \%$ & Delgado (O) & $6 \%$ & Maiz del cerro $(T)$ & $6 \%$ & & \\
\hline Corto amarillo $(\mathrm{O})$ & $2 \%$ & Veracruzano $(T x)$ & $6 \%$ & Tardón (O) & $3 \%$ & & \\
\hline Morado (Tx) & $2 \%$ & Largo grande (Tx) & $3 \%$ & & & & \\
\hline Olotillo amarillo (O) & $2 \%$ & Maíz de camarón $(\mathrm{Tx})$ & $3 \%$ & & & & \\
\hline Olotillo maiz boludo $(0)$ & $2 \%$ & Conejo (C) & $3 \%$ & & & & \\
\hline Olotillo maíz delgado $(\mathrm{O})$ & $2 \%$ & Morado $(\mathrm{Zg})$ & $3 \%$ & & & & \\
\hline Olotillo medio largo (C) & $2 \%$ & Olotillo largo (O) & $3 \%$ & & & & \\
\hline Pinto $(T x)$ & $2 \%$ & Palmasol (Tx) & $3 \%$ & & & & \\
\hline Ratita (O) & $2 \%$ & & & & & & \\
\hline Siete color $(\mathrm{Tx})$ & $2 \%$ & & & & & & \\
\hline Total & $100 \%$ & & $100 \%$ & & $100 \%$ & & $100 \%$ \\
\hline
\end{tabular}

*Race primaire: $\mathrm{O}=$ Olotillo, $\mathrm{Tx}=$ Tuxpeño, $\mathrm{C}=$ Conejo, $\mathrm{T}=$ Tepecintle, $\mathrm{P}=$ Pepitilla, $\mathrm{Zp}=$ Zapalote grande . Pour chaque type de maiis s'indique uniquement la race primaire plus commune de ce type de maïs

Dans la zone chatine, les agriculteurs utilisent principalement les caractères morphologiques du maïs pour définir le nom (Tableau 5). En Ch-M nous pouvons classer les types de maïs en deux grandes catégories : l'olotillo et les autres (Figure 3). Nous employons " autres » car, dans la communauté, il y a une catégorie non dénommée pour les maïs qui ne sont pas olotillo. Olotillo est également une race de maïs reconnue botaniquement et amplement cultivée dans les zones basses tropicales du Mexique (Wellhausen et al. 1952). Dans la catégorie olotillo on utilise les caractères de l'épi pour différencier les types, par ordre d'importance, ce sont: la couleur, le temps de maturation et la forme du grain. Par rapport à la couleur, on distingue deux types l'olotillo blanc et l'olotillo jaune. En ce qui concerne le temps de maturation, les types sont olotillo corto (précoce) et olotillo largo (intermediaire-longue). Nous avons trouvé qu'il existe trois niveaux de classification à $\mathrm{Ch}-\mathrm{M}$, davantage que dans les autres localités où il n'y en a que deux (Figure 3). La première division est entre l'olotillo et les autres, ensuite par les cycles des olotillo courts et longs, et finalement on classe par la couleur ou la forme du grain. 
Figure 3 : Structure de la classification du maïs dans chaque localité

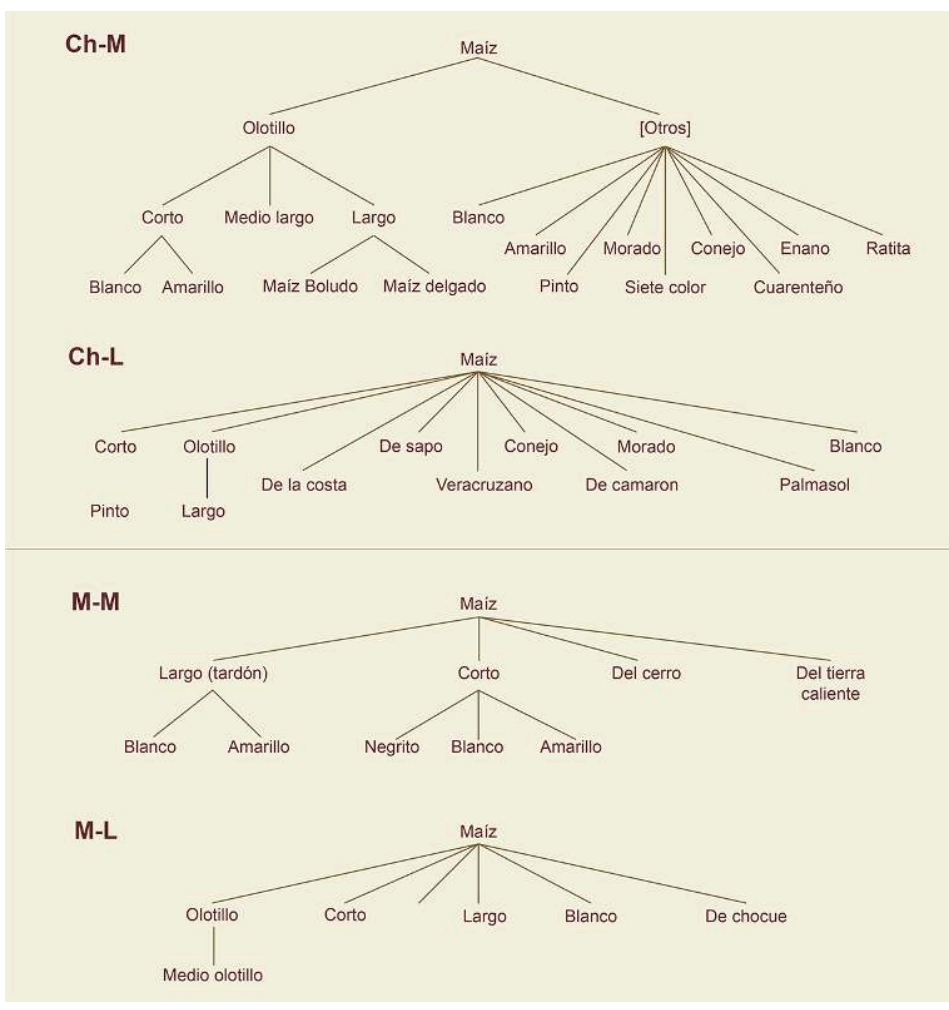

Dans cette même localité, les autres types de maïs qui ne sont pas olotillo, sont nommés par leurs caractères plus remarquables ou distinctifs du type de maïs. La couleur est l'un des caractères, ainsi les types: amarillo, blanco, pinto, morado et siete colores (épis dont les grains mélangent sept couleurs). Il se peut que d'autres caractères soient employés pour différencier mieux une variété, par exemple, amarillo corto, jaune précoce. D'autres types importants sont le maïs cuarenteño, une variété à croissance rapide qui en 40 jours passe de la semence à la floraison; le maïs enano, est une plante basse ; le conejo est similaire au cuarenteño en ce qui concerne son temps de maturation, mais l'épi est plus fin. L'exception à la règle est le type de maïs ratita, pour lequel l'agricultrice n'a pas pu donner une explication fondée sur la morphologie de l'épi ou de la plante ; elle a simplement dit que c'était la façon dont sa grand-mère l'appelait.

À Ch-L, actuellement le maïs le plus important est le maïs sapo, crapaud, $20 \%$ des échantillons correspondent à ce type, le nom est issu de la forme de l'épi, qui est grosse et petite, semblable au crapaud. Ce type de maïs est précoce. À Ch-L, il y a un autre type de maïs précoce appelé corto, il est moins précoce que les types cuarenteño et conejo trouvés ailleurs.

Dans cette localité, l'olotillo n'est pas aussi important qu'à Ch-M; là, les agriculteurs nomment différemment l'olotillo, c'est l'olote delgado. Si nous additionnons les pourcentages d'olotillo et d'olote delgado, ces types de maïs correspondent à $26 \%$ des échantillons collectés dans la localité (Tableau 5). À Ch-L, on utilise également le lieu d'origine pour nommer les types de maïs et dans ces cas les noms ne dénotent pas des caractères de l'épi, tels que les maïs de la costa et le veracruzano, tous deux ressemblent par leur forme au maïs sapo. Selon les agriculteurs, d'autres maïs s'utilisent de moins en moins, tels que le maïs camarón et le palmasol. Le premier a de petits épis, mais non pas gros comme le sapo. Le second est de type tardif, qui produit de grands épis gros et 
maintenant on préfère les maïs de cycle moyen. Ces maïs ont été déplacés parce que le sapo est plus productif. Dans cette localité, on classe le maïs au maximum en deux niveaux hiérarchiques, et les seules catégories qui se subdivisent sont corto et olotillo. La plupart des variétés ne sont pas groupées dans des catégories hiérarchisées (Figure 3).

Dans les localités mixtèques, les critères des noms des maïs sont: le temps de maturation, la couleur de l'épi, la zone d'adaptation et la forme de l'épi. Dans la localité $\mathrm{M}-\mathrm{M}$, les deux catégories les plus générales sont le temps de maturation, dont les types de maïs communs sont : largo ou tardón (cycle long-moyen) et corto (précoce). Le maïs corto connaît trois variétés définies par la couleur du grain: blanc, jaune et noir (Figure 3). Le blanc est le type le plus commun et généralement les agriculteurs disent simplement corto pour dire corto blanco. Si nous regroupons les trois couleurs du corto, il représente $42 \%$ des échantillons collectés dans la localité. Ces types de maïs appartiennent à la race Conejo, c'est dans cette localité que nous avons collecté la plus grande quantité de cette race. Le type blanco est à cycle long, mais se différencie du largo parce que la couleur est définitivement blanche. L'amarillo est un maïs à cycle moyen, et se caractérise par sa couleur jaune foncé (Tableau 5).

Le maïs de tierra caliente est un type de maïs qui est semé dans les zones basses, même si la localité est située dans la partie haute de la municipalité, certains agriculteurs descendent dans les basses terres pour le semer. Le maïs del cerro, du coteau, est semé dans les parties hautes où dominent les forêts de chêne. Ce type de maïs est à cycle long, mais se différencie du largo, car il est adapté aux terres hautes.

26 À M-L, en comparaison avec les autres communautés, on trouve le nombre le plus réduit des noms de maïs. Les quatre premiers types, les plus communs, ont un pourcentage de collecte semblable, montrant plus d'équité que dans les trois autres localités (Tableau 5.) Dans cette localité, les agriculteurs reconnaissent que l'olotillo s'est mélangé, c'est pourquoi ils le nomment un medio olotillo, olotillo moyen; il est une catégorie du précédent (Figure 3). Là et dans d'autres localités, certains agriculteurs le nomment maïs olotillo, même si les principaux caractères ont changé. Aujourd'hui, l'épi est plus gros et le cœur de l'épi est plus épais, ce qui indique le mélange avec des types de maïs de la race Tuxpeño. Ceci est dû au fait qu'à chaque occasion, les agriculteurs ont sélectionné des épis plus épais, mais étant donné qu'à l'origine leur maïs était olotillo, ils ont continué à l'appeler ainsi.

Nous avons retrouvé des noms uniques dans chaque localité et d'autres partagés (Figure 4). Dans les localités chatines, nous avons retrouvé plus de noms uniques, c'està-dire qui ne sont pas utilisés ailleurs. À Ch-M, les noms exclusifs de cette localité sont : cuarenteño, maíz enano, olotillo amarillo, olotillo corto, olotillo maíz boludo, rondouillard, olotillo maíz delgado, siete color, ratita et pinto, mauve. Dans la localité de Ch-L, les noms exclusifs sont : corto pinto, maíz de camarón, largo grande, de la costa, palmasol, veracruzano, de Vera Cruz et maíz de sapo. Il y a peu de noms communs entre les localités et les municipalités. Blanco est le seul nom qui est commun aux quatre localités. Corto et olotillo sont partagés dans trois localités, le type olotillo n'a pas été retrouvé dans la localité de $\mathrm{M}-\mathrm{M}$, mais lors des entretiens avec les agriculteurs, ils se sont souvenus $\mathrm{du}$ nom et s'y sont référés comme chirique. 
Figure 4 : Noms locaux des types de maïs dans les quatre localités

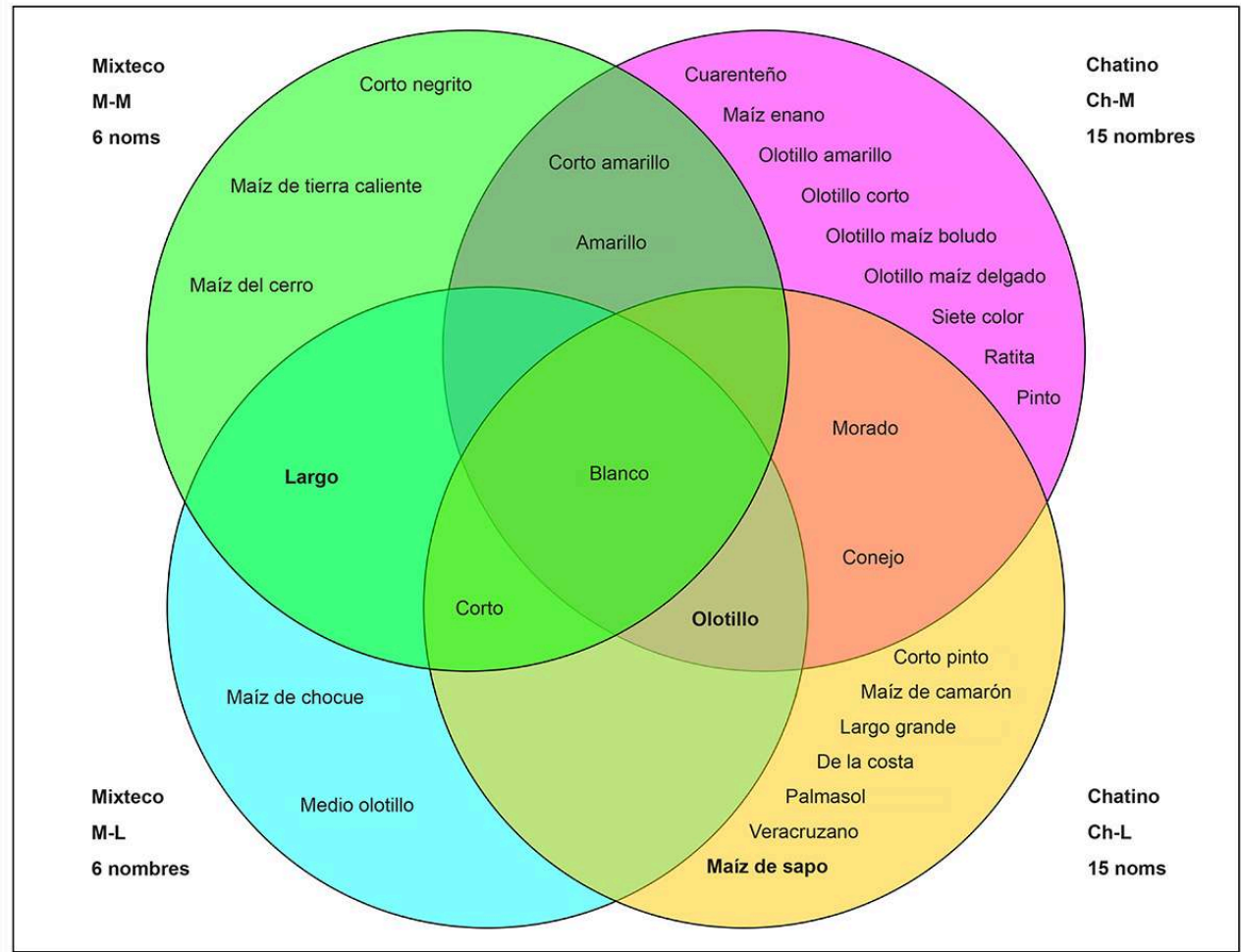

Dans certaines localités, des types de maïs de caractères semblables portent des noms différents ; par exemple, le type corto des localités mixtèques est appelé cuarenteño, et dans des localités chatines, conejo. Le maïs sapo de la localité Ch-L est semblable au maïs enano de Ch-M. Il existe aussi des noms similaires entre localités, mais qui font référence à des types différents; par exemple, l'olotillo de Ch-M est différent de l'olotillo de M-L et le corto de M-M est différent du corto de Ch-L. Même si toutes les races sont présentes dans les populations de maïs des deux municipalités, ce sont les proportions qui varient. L'olotillo domine entre les maïs des Chatines, particulièrement dans la localité Ch-M ; alors que le conejo est le plus représentatif dans les milpas mixtèques, particulièrement à $\mathrm{M}-\mathrm{M}$ (Tableau 4). Cette présence majoritaire de races ne s'exprime pas en nombres de collectes, mais en nombre de noms locaux et en nombre de variantes. Par exemple, à Ch-M, le type olotillo a sept variantes et à $\mathrm{M}-\mathrm{M}$ le type corto (race conejo) a trois variantes (Tableau 5).

\section{Discussion}

Le savoir des agriculteurs, à propos de la diversité des maïs de leur région, est grand. Ceci inclut les niveaux d'adaptation des variétés, l'utilité et la nécessité des divers types de maïs, ainsi que les changements qui ont eu lieu au cours des dernières années. Ainsi comme Hernández-Xolocotzi (1972) l'avait signalé, les agriculteurs et leur classification des maïs doivent être le point de départ pour toute recherche sur la diversité régionale. Nos résultats sur les noms locaux et la variabilité entre les localités sont semblables à ceux d'autres études (Sadiki et al. 2007), dans le sens qu'il existe des noms différents pour des types de maïs avec des caractères d'épis semblables. Dans notre cas, ceci arrive même dans des localités du même groupe ethnique, par exemple, le type olotillo 
de Ch-M, est appelé olote delgado à Ch-L et chiriqui à M-M. Celui qui est nommé corto à M$\mathrm{M}$, s'appelle conejo à Ch-L. De même, les types de maïs avec le même nom correspondent à différents cultivars. Par exemple, le corto à $\mathrm{M}-\mathrm{M}$, qui correspond à la race Conejo, est distinct du corto à M-L qui est de la race Tuxpeño et du corto de Ch-L qui est de la race olotillo. Grâce à leur expérience, la communication entre des agriculteurs du même groupe ethnique est possible, car ils connaissent les noms équivalents des maïs entre les différentes localités. Toutefois, la communication entre des agriculteurs des différents groupes ethniques est plus difficile, car ils n'utilisent pas le même nom, et ne connaissent pas le nom équivalent. Un autre fait qui rend la communication difficile entre des localités est que le nom de la variété la plus commune ne se partage pas entre les trois localités : olotillo, à Ch-M, maíz de cerro à M-M et maíz de sapo à Ch-L.

Une des questions que les chercheurs sur l'agrobiodiversité se sont posée est de connaître le nombre des variétés locales comme indicateur de la diversité génétique (Sadiki et al. 2007 ; Jarvis et al. 2008). Dans cette étude, les noms locaux comparés avec les données de diversité génétique basées sur des microsatellites (Orozco-Ramírez et al. 2016), montrent qu'une telle relation n'existe pas entre les localités étudiées. La localité avec la plus grande diversité génétique fondée évaluée sur les marqueurs moléculaires $\mathrm{SSR}^{5}$ est M-M et c'est la troisième localité en quantité de noms. La localité avec le plus de noms se trouve en deuxième place pour la diversité génétique. Toutefois, l'existence de noms différents, se référant aux variétés de la même race ou qui ne montrent pas de différences génétiques perceptibles importantes, a une signification et une utilité pour les agriculteurs. La diversité des maïs perçue par les agriculteurs est supérieure du point de vue agronomique, une même race peut être formée par plusieurs variétés locales. Dans notre cas, la classification locale est plus importante que la diversité génétique trouvée en utilisant les marqueurs moléculaires, car les variétés d'une localité ne sont pas discernables en employant des microsatellites (Orozco-Ramírez et al. 2016).

Lorsque les classifications de maïs de quatre localités sont comparées, on trouve des similitudes et des différences (Figure 3). Premièrement, comme cela a été décrit dans d'autres publications (p. ex. Bellon \& Brush 1994), la classification se fonde sur des caractéristiques de morphologie de l'épi, du cycle de vie, de l'adaptation et de l'origine de la semence. Pourtant, les catégories qui définissent la structure hiérarchique de la classification montrent des variations entre les localités. La plus grande différence est entre les localités de M-M et Ch-M. Dans la première, les catégories du cycle de la culture (courte vs. longue) et le contexte de la culture (del cerro vs. de tierra caliente) définissent la structure taxonomique. Alors que dans la localité $\mathrm{Ch}-\mathrm{L}$, le groupe olotillo est clé dans la classification. Ceci se rapproche des propositions selon lesquelles la culture influe sur les classifications traditionnelles (López et al. 1997). Plus une culture est connue, plus la classification locale est détaillée (Boster 1986). En regardant les différences hiérarchiques des classifications, et la basse fréquence de noms partagés, surtout des types de maïs les plus courants dans chaque localité, nous supposons qu'il y a peu de motivations pour que les agriculteurs d'un groupe ethnique cherchent des semences dans d'autres groupes ethniques des localités voisines, étant donné qu'ils n'y trouvent pas de maïs qu'ils connaissent.

La variation du nombre de noms locaux de maïs entre localités a plusieurs implications. Une classification locale plus détaillée, avec un plus grand nombre des noms, pourrait indiquer une connaissance plus profonde de la diversité des maïs. Boster (1986) qui a 
comparé la classification du manioc entre hommes et femmes, a trouvé que les femmes ont un savoir plus profond et sont capables de nommer plus de variétés car elles sont les principales responsables de la culture. De même, une liste avec plus de noms peut aussi signaler qu'il y a peu d'échange de variétés entre les localités. Ceci aura pour conséquence le maintien d'un plus grand nombre de maïs à l'intérieur de la famille. Ceci parce que le nom local, est l'information de base dont un agriculteur a besoin dans le processus d'acquisition de semences (Badstue et al. 2007).

Nous supposons que les échanges de semences dans une localité ne sont pas communs, il n'y a donc pas de nécessité d'homogénéiser des noms qui font référence aux types de maïs similaires. Nous supposons aussi que chaque famille peut maintenir un nom particulier pour des variétés communes. Ceci pourrait expliquer l'existence de différents noms dans la localité de Ch-L pour les types de maïs dont les caractéristiques sont partagées, par exemple : maíz de camarón, maíz de sapo, veracruzano et maíz de la costa, qui sont associés à la région côtière. D'un autre côté, un moindre nombre de noms locaux, comme c'est le cas danns la localité M-L, est associé au fait qu'il existe moins de terres disponibles pour la culture du maïs, donc une moindre quantité d'agroenvironnements. Comme nous l'avons montré dans les résultats, pour le groupe mixtèque, la zone de culture du maiis est l'une des catégories la plus générale pour la classification de maïs (maíz de cerro vs. maíz de tierra caliente).

Il manque encore plus des recueils de variétés locales et de leurs noms en langues amérindiennes pour essayer de reconstituer la dispersion régionale des variétés. L'information linguistique et génétique sur les maiis à l'échelle régionale permet de poser l'hypothèse, à partir de la dispersion régionale des peuplements chatines, que les Chatines ont migré de la côte vers le nord et nord-est, à cause de l'invasion espagnole qui s'est approprié les plateaux proches de la mer (Campbell 2014). Ce type de recherche pourrait permettre de savoir s'ils ont migré avec leur maïs ou s'ils ont acquis de nouvelles variétés dans les communautés agricoles voisines. Avec les données recueillies sur le vocabulaire des parties du maïs, nous avons trouvé qu'il existe des similitudes à l'intérieur des groupes linguistiques mixtèques et chatines, alors qu'il y a d'importantes différences entre ces deux groupes. Ceci peut s'expliquer par la séparation ancienne des langues (Kaufman 1990), qui ne permet pas un flux d'information entre les deux langues, ce que confirment les échanges réduits de semences entre agriculteurs de groupes ethniques différents, même lorsqu'ils résident dans des localités voisines.

\section{Conclusions}

La diversité et la séparation des populations de maïs entre les municipalités d'Amoltepec (Mixtèque) et Zenzontepec (Chatine) se maintiennent et sont renforcées par la séparation culturelle et linguistique. Dans la terminologie et la structure de la classification du maïs, les Chatines et les Mixtèques se distinguent, même dans des localités proches, et ont des caractéristiques agronomiques et d'organisation sociale similaires. Cette séparation est renforcée par le manque de moyens de communication entre les deux municipalités et par l'histoire de conflit. Le vocabulaire varié et spécifique du maïs, nous montre qu'il est un sujet important dans la vie de ces communautés amérindiennes. De plus, la riche nomenclature de la diversité du maïs montre d'un côté, que les agriculteurs sont conscients de la diversité qu'ils cultivent ; et 
d'un autre côté, que la diversité s'explique par des caractéristiques écologiques et d'utilisation pratique. Ainsi, il reste à associer cette diversité aux valeurs symboliques de chaque communauté amérindienne.

Nous remercions les agriculteurs des communautés de Santiago Amoltepec et Santa Cruz Zenzontepec d'avoir participé à cette recherche, tout particulièrement Fabio Velasco pour son aide pendant le terrain, le professeur Alfonso Merino et le professeur Margarito García pour leur aide sur le vocabulaire du maïs. M.C. Flavio Aragón pour son aide lors de l'identification des races. Le premier auteur remercie le CONACYT et l'UC MEXUS pour le financement de la recherche et aussi le Centre de Recherche de Géographie environnementale de l'UNAM (Université Nationale) pour la bourse de post-doctorat qui a permis la rédaction de cet article.

\section{BIBLIOGRAPHIE}

Anderson E. 1946 - Maize in México: A preliminary survey. Annals of the Missouri Botanical Garden 33 (2) :147-247.

Aragón-Cuevas F., Figueroa-Cárdenas J., Flores-Zarate M., Gaytán-Martínez M. \& Véles Medina J.J. 2012 - Calidad industrial de maíces nativos de la sierra sur de Oaxaca. Oaxaca, Instituto de Investigaciones Forestales Agricolas y Pecuarias, $249 \mathrm{p}$.

Badstue L.B., Bellon M.R., Berthaud J., Ramirez A., Flores D. \& Juarez X. 2007 - The Dynamics of Farmers' Maize Seed Supply Practices in the Central Valleys of Oaxaca, Mexico. World Development 35 (9) : 1579-1593.

Bellon M.R. \& Brush S.B. 1994 - Keepers of maize in Chiapas, Mexico. Economic Botany 48 (2) : 196-209.

Berlin B. 1992 - Ethnobiological classification: principles of categorization of plants and animals in traditional societies. Princeton, Princeton University Press, 354 p.

Beaty de Farris K. 2004 - Diccionario básico del Mixteco de Yosundúa Oaxaca. Segunda edición. México DF, Instituto Lingüístico de Verano. 175 p.

Boster J.S. 1986 - Requiem for the omniscient informant: there's life in the old girl yet. In : Dougherty J. (Ed) Explorations in cognitive anthropology. Urbana, Illinois, University of Illinois Press : $177-197$.

Campbell S.S., Peterson A.J. \& Lorenzo Cruz F. 1986 - Diccionario Mixteco de San Juan Colorado. México. DF. Instituto Lingüístico de Verano, 209 p.

Campbell E.W. 2014 - Aspects of the phonology and morphology of Zenzontepec Chatino, a Zapotecan language of Oaxaca, Mexico. Doctorado en Lingüíistica, Austin, University of Texas at Austin, 381 p. Chávez-Servia J.L., Diego-Flores P. \& Carrillo-Rodríguez J.C. - Complejos raciales de poblaciones de maíz evaluadas en San Martín Huamelulpan, Oaxaca. Ra Ximhai 7 (1) :107-115.

Hernández Xolocotzi E. 1972 - Exploración etnobotánica en maíz. Fitotecnia latinoamericana 8 (2) : 46-51. 
Hopkins N. 1984 - Otomanguean linguistic prehistory. In : Josserand K., Winter M. \& Hopkins N. (Ed.) Essays in Otomanguean culture history. Nashville: Vanderbilt University : 25-64.

Hill J.H. 2006 - The historical linguistics of maize cultivation in Mesoamerica and North America. In : Staller J., Tykot R. \& Benz B. (Ed.), Histories of maize: multidisciplinary approaches to the prehistory, linguistics, biogeography, domestication, and evolution of maize. San Diego, CA, Elsvier, et Burlington, Massachusetts, Academic Press : 631-645.

INALI 2009 - Catalogo de las lenguas indigenas nacionales, variantes lingüisticas de México con sus autodenominaciones y referencias geoestadísticas. Mexico, Instituto Nacional de Lenguas Indígenas, $260 \mathrm{p}$.

INEGI 2010 - Censo de poblacion y vivienda 2010, principales resultados por localidad. México, Instituto Nacional de Estadísitca y Geografía. http://www.inegi.org.mx/sistemas/consulta_resultados/ iter2010.aspx [Consulté 22 mars 2013].

INEGI 2013 - Conjunto de datos vectoriales de la serie topográfica y de recursos naturales escala 1:1 000 000. México, Institituo Nacional de Estadísitca y Geografía. http://www.inegi.org.mx/geo/ contenidos/recnat/default.aspx [Consulté 22 mars 2013].

Jarvis D.I, Brown A.H.D., Cuong P.H., Collado-Panduro L., Latournerie-Moreno L., Gyawali S., Tanto T., Sawadogo M., Mar I. \& Sadiki M. 2008 - A global perspective of the richness and evenness of traditional crop-variety diversity maintained by farming communities. Proceedings of the National Academy of Sciences 105 (14) : 5326-5331.

Júnior W.S.F., Gonçalves P.H.S., de Lucena R.F.P. \& Albuquerque U.P. 2016 - Alternative Views of Folk Classification. In : Albuquerque U.P., Alves R.R.N. (Ed.), Introduction to Ethnobiology. Switzerland, Springer International Publishing : 123-128.

Kaufman T. 1990 - Early Otomanguean Homelands and Cultures: Some Premature Hypotheses. University of Pittsburgh Working Papers in Linguistics 1 : 91-136.

López A., Atran S., Coley J.D., Medin D.L. \& Smith E.E. 1997 - The tree of life: Universal and cultural features of folkbiological taxonomies and inductions. Cognitive psychology 32 (3) :251-295.

Orozco-Ramírez Q. 2014 - Maize Diversity and Population Structure Related to Ethno-Linguistic Variation. PhD Dissertation, Davis, USA. University of California, $286 \mathrm{p}$.

Orozco-Ramírez Q., Ross-Ibarra J., Santacruz-Varela A. \& Brush S. 2016 - Maize diversity associated with social origin and environmental variation in Southern Mexico. Heredity 116 (5) : 477-484.

Pride K \& Pride L. 2010 - Diccionario Chatino de la zona alta, Panixtlahuaca Oaxaca, y otros pueblos. México DF, Instituto Lingüístico de Verano, 483 p.

Pride L. \& Pride K. 1970 - Vocabulario chatino de Tataltepec. Mexico DF, Instituto Lingüístico de Verano, $103 \mathrm{p}$.

Sadiki M., Jarvis D., Rijal D., Bajracharya J., Hue N., Camacho-Villa T., Burgos-May L., Sawadogo M., Balma D., Lope D. et al. 2007 - Nombres de las variedades. In : Jarvis D., Padoch C. \& Cooper H.D. (Ed.), Manejo de la Biodiversidad en los Ecosistemas Agrícolas. Bioversity International : 37-81.

Sánchez J., Goodman M. \& Stuber C. 2000 - Isozymatic and morphological diversity in the races of maize of México. Economic Botany 54 : 43-59.

Stross B. 2006 - Maize in Word and Image in Southeastern Mesoamerica. In : Staller J., Tykot R. \& Benz B. (Ed.), Histories of maize: multidisciplinary approaches to the prehistory, linguistics, biogeography, 
domestication, and evolution of maize. San Diego, CA, Elsvier, et Burlington, Massachusetts, Academic Press : 577-598.

Wellhausen E., Roberts J., Roberts L. M. \& Hernández X. E. 1952 - Races of maize in Mexico, their origin, characteristics, and distribution. Cambridge, MA, The Bussey Institution, Harvard University, $223 \mathrm{p}$.

Zurita-Benavides M.G. 2016 - Choix paysanne des variétés de maïs et agrobiodiversité. Paris, Editions universitaires européennes, $136 \mathrm{p}$.

\section{NOTES}

1. Ce terme désigne des parents par la consanguinité; en végétation, cela désigne, des descendants directs et proches.

2. Les Chatines (chatinos) sont un peuple indigène de l'État de Oaxaca qui se dénomment euxmêmes kitse cha'tnio, et parlent la langue cha'cña.

3. Les noms des races de maïs sont écrits avec des majuscules et les noms locaux en italique pour les types de maïs.

4. Mot aztèque qui désigne l'enveloppe de l'épi de maïs. (note de FAS)

5. SSR: Simple Sequence Repeats "Répétitions de séquences simples», un type de marqueur moléculaire

\section{RÉSUMÉS}

Cet article explore le savoir local de la diversité du maïs de deux communautés amérindiennes. Nous décrivons les différences du vocabulaire du maïs en langue et classification locales pour montrer comment ces populations différencient leurs maïs. Nous avons étudié deux municipalités voisines de la Sierra Sur Oaxaca, l'une habitée par des Mixtèques et l'autre par des Chatines. Cette approche comparative de deux municipalités a compris également les caractères environnementaux et culturels ainsi que les savoirs sur la diversité des maïs. Nous avons eu des entretiens et collecté des échantillons de maïs dans deux localités de chaque municipalité dont les caractères environnementaux sont opposés. La classification de la diversité des maïs à travers leurs noms locaux montre des différences structurales et de richesse, tant entre localités qu'entre municipalités. Les noms locaux ainsi que la distribution des races sont différents entre les municipalités. On sait bien que le vocabulaire du maïs dans les langues amérindiennes est varié et complexe, mais nous n'avons pas retrouvés les cognats ${ }^{1}$ des types de maïs. Ces résultats nous amèneront à la conclusion que la diversité et la distinction des populations de maïs entre les municipalités d'Amoltepec (Mixtèque) et de Zenzontepec (Chatine) se maintient et se renforce par la séparation culturelle et linguistique.

This paper explores the traditional knowledge about maize diversity in two indigenous communities. We describe the differences on maize vocabularies and on the folk taxonomy in order to define their effect on maize population differentiation between both indigenous communities. We work in two neighboring municipalities located in the Sierra Sur, Oaxaca, one Mixtec and the other Chatino. We use a comparative approach to describe the environment and 
the culture of both municipalities. We link the local maize knowledge to its diversity, looking at local maize classification and maize vocabulary. In both municipalities, we performed interviews and maize collections in two villages with contrasting environment. Classification of maize diversity by local name shows differences in structure and in richness, from one locality to the other and from one municipality to the other. We found differences between municipalities in both the list of local names and the maize race distribution. Maize vocabulary is rich and complex among both Chatino and Mixtec farmers, we did not find maize-type cognates. Following those results, we concluded that maize diversity and maize population differentiation between Mixtec and Chatino municipalities is maintained and reinforced by the cultural and linguistic separation.

Este artículo explora el conocimiento local de la diversidad del maíz en dos comunidades indígenas. Describimos las diferencias de los vocabularios del maíz en lengua local y de la clasificación local con el fin de dilucidar su efecto en la diferenciación de las poblaciones de maíz que ambos grupos manejan. Trabajamos en dos municipios vecinos de la Sierra Sur, Oaxaca, uno habitado por hablantes de mixteco y el otro de chatino. Se utilizó un enfoque comparativo para describir el ambiente y la cultura de los dos municipios y se hace un vínculo con el conocimiento de la diversidad del maíz. Se realizaron entrevistas y se colectaron maíces en dos localidades de cada municipio ubicadas en ambientes contrastantes. La clasificación de la diversidad del maíz a través de los nombres locales muestra diferencias estructurales y de riqueza, tanto entre localidades como entre municipios. Encontramos que tanto los nombres locales como la distribución de las razas son diferente entre municipios. El vocabulario del maíz en las lenguas indígenas es variado y complejo, no encontramos cognados de los tipos de maíz. A partir de estos resultados concluimos que la diversidad y la separación de las poblaciones de maíz entre los municipios de Amoltepec (mixteco) y Zenzontepec (chatino) se mantienen y fortalecen por la separación cultural y lingüística.

\section{INDEX}

Index géographique : Mexique

Mots-clés : Mixtèque, Chatine, maïs indigène, classification locale, langues amérindiennes

Palabras claves: Mixteco, Chatino, Maíces nativos, Clasificación local, Lenguas indígenas

Keywords : Mixtec, Chatino, Native maize, Local classification, Indigenous languages

\section{AUTEURS}

\section{QUETZALCÓATL OROZCO-RAMÍREZ}

Instituto de

Geografia, UNAM. Reforma S/N Centro,

Oaxaca, Oaxaca, Mexico qorozco@gmail.com

\section{STEPHEN BRUSH}

Professeur Emérite, Department of Human Ecology, University of California. Davis, California, USA 